\title{
Flipping a Coin? Predicting Nodal Status After Neoadjuvant Chemotherapy
}

\author{
Theresa Schwartz, MD, MS, FACS ${ }^{1}$ and Julie Margenthaler, MD, FACS ${ }^{2}$ \\ ${ }^{1}$ Department of Surgery, Saint Louis University School of Medicine, St. Louis, MO; ${ }^{2}$ Department of Surgery, Washington \\ University in Saint Louis, St. Louis, MO
}

Axillary management for patients with breast cancer continues to evolve. Less than 10 years ago, the standard of care for the patient with clinically node-positive disease was an axillary lymph node dissection (ALND). As the use of neoadjuvant chemotherapy (NAC) has broadened, so has our curiosity regarding appropriate nodal staging after systemic treatment. Upfront sentinel lymph node biopsy (SLNB) was initially recommended for the clinically nodenegative patient before NAC due to concern for an unacceptably high false-negative rate if performed after therapy.

With the unclear effect of NAC on the lymphatic drainage patterns of the breast, the reliability of SLNB after NAC was called into question. ${ }^{1,2}$ However, the feasibility and accuracy of SLNB after NAC has been demonstrated in multiple studies, including National Surgical Adjuvant Breast and Bowel Project (NSABP) B-27. ${ }^{3}$ This has expanded the use of NAC not only to breast cancer patients with larger tumors who wish to be lumpectomy candidates, but also to those with initially clinically node-positive disease with the hopes of minimizing the need for an ALND. The optimal technique for performing SLNB on the initially clinically node-positive patient after NAC, comprising patient selection, pretreatment imaging, a biopsy clip in the lymph node before treatment, posttreatment imaging, type of tracer used, method of histologic analysis, has not been uniformly defined to date.

(C) Society of Surgical Oncology 2019

First Received: 20 August 2019;

Published Online: 25 September 2019

T. Schwartz, MD, MS, FACS

e-mail: theresa.schwartz@health.slu.edu
In this issue of Annals of Surgical Oncology, Moo et al. ${ }^{4}$ compares the accuracy of axillary clinical exam with that of breast magnetic resonance imaging (MRI) in predicting axillary nodal response to NAC for the initially clinically node-positive patient. Using their single-center prospectively maintained NAC database, they identified 269 studyeligible breast cancer patients with clinical T0-T3 N1 disease who underwent NAC followed by definitive breast surgery and SLNB between March 2014 and July 2017. All the patients had pathologically confirmed axillary nodal metastases before NAC and no palpable axillary lymph nodes on the clinical exam after NAC. Axillary lymph nodes were considered abnormal on post-NAC MRI if they had a thickened $(>4 \mathrm{~mm})$ or irregular cortex, appeared rounded, or had loss of a fatty hilum. Consistent with their previous studies, ${ }^{5}$ a sentinel node was considered positive if metastasis of any size was identified, including isolated tumor cells, and immunohistochemical staining was used only when hematoxylin and eosin (H\&E) could not indicate a definitive diagnosis.

Of the 182 patients who underwent a post-NAC MRI, 55 (30\%) had abnormal-appearing lymph nodes on MRI, and $32(58 \%)$ of these patients had a positive SLNB. Among the 127 patients with normal-appearing lymph nodes on post-NAC MRI, 53 (42\%) ultimately had a positive SLNB. Among the 87 patients who had a negative clinical axillary exam only and did not undergo a post-NAC MRI, 45 (52\%) had a positive SLNB. After calculating the overall accuracy of post-NAC MRI in predicting SLNB status to be $58 \%$, compared with an accuracy of $48 \%$ for a clinical exam alone, the authors concluded that post-NAC MRI has limited benefit in predicting nodal involvement.

Since 2005, when SLNB became the gold standard for axillary staging of the clinically node-negative patient with breast cancer, we have been searching for the least invasive 
method to determine its eligibility. Before this, preoperative assessment of the axilla outside of a clinical exam was seemingly unnecessary because all patients underwent an ALND for definitive nodal staging. Findings have repeatedly shown that a clinical axillary exam is unreliable because more than $30 \%$ of patients with a normal axillary exam are found to have nodal metastases on surgical pathology. ${ }^{6-8}$ A clinically positive axilla is not always accurate either, with a reported positive predictive value (PPV) of $77 \%$ to $84 \%$ in experienced hands. ${ }^{8,9}$

Breast MRI was developed in the mid-1980s, and its frequency of use increased with the advent of NAC protocols in the 1990s. Quite often, breast MRI was performed both before and after NAC to assess treatment response and plan surgical intervention. Although the main focus of breast MRI was on the primary tumor, a large number of studies investigated the use of MRI in evaluating the axillary nodal basin and predicting nodal status. However, the majority of these studies had small samples with limited power. Additionally, they had different subjective and/ or reference standards to describe the appearance of an abnormal lymph node.

With these inconsistencies, the MRI characteristics of a lymph node suspicious for containing metastatic disease have not been uniformly defined in the literature. ${ }^{10-16}$ Thickened cortex, ${ }^{10,11,13,14,16}$ irregular cortex, ${ }^{11,14,16}$ and loss of fatty hilum ${ }^{14-16}$ are among the most common MRI characteristics deemed to be associated with nodal positivity, but none have a specificity or sensitivity high enough to make them predictive without histologic confirmation.

During the past 40 years, the practice-changing literature on breast cancer has provided data and guidelines to allow for deescalation of our surgical treatment with no change in survival outcomes. Investigation of less extensive and less invasive techniques was warranted because the number and severity of adverse treatment effects were becoming more common and more pronounced.

Management of the axilla in the clinically node-positive patient is the next hurdle to jump. With three multicenter, prospective, clinical trials investigating the utility of SLNB for such patients, it is of primary importance that we identify and agree on the assessment strategies needed to determine which patients can undergo SLNB and which should proceed to ALND. Ultimately, we may be told whether we need to do any further histologic evaluation of the axilla.

As Moo et al. ${ }^{4}$ have outlined in their report, MRI alone is not adequate for predicting nodal status after neoadjuvant chemotherapy. Breast cancer management requires a team effort, and in very few instances is one test or assessment enough for creation of a treatment plan. The multidisciplinary approach to implementing all forthcoming data should improve our odds of predicting nodal status.

We argue that determining what preoperative study to perform and the method or need for intraoperative nodal assessment after NAC should not be the ultimate research focus for this cohort of patients. Our focus should be on how to decide whether we should proceed with a completion ALND in the setting of a positive SLNB after NAC. Prior data demonstrate that even low-volume residual disease in the sentinel lymph node (SLN) or SLNs after NAC is associated with a significantly higher risk of additional positive axillary lymph nodes. ${ }^{17}$ Does completion ALND in the setting of a positive SLNB after NAC translate to decreased locoregional recurrence or improved survival? That is the question we need to answer to optimize outcomes and limit morbidity for our patients. This question cannot be decided by flipping a coin.

\section{REFERENCES}

1. Tausch C, Steger GG, Haid A, Jakesz R, Fridrik MA, Reitsamer $\mathrm{R}$, et al. Sentinel node biopsy after primary chemotherapy in breast cancer: a note of caution from results of ABCSG-14. Breast J. 2001;17:230-8.

2. Pecha V, Kolarik D, Kozevnikova R, Hovorkova K, Hrabetova P, Halaska M, et al. Sentinel lymph node biopsy in breast cancer patients treated with neoadjuvant chemotherapy. Cancer. 2011;117:4606-16.

3. Mamounas EP, Brown A, Anderson S, Smith R, Julian T, Miller $B$, et al. Sentinel lymph node biopsy after neoadjuvant chemotherapy in breast cancer: results from National Surgical Breast and Bowel Project Protocol B-27. J Clin Oncol. 2005;23:2694-702.

4. Moo TA, Jochelson M, Zabor E, et al. Is clinical exam of the axilla sufficient to select node-positive patients who downstage after NAC for SLNB? A comparison of the accuracy of clinical exam vs MRI. Ann Surg Oncol. 2019. https://doi.org/10.1245/ s10434-019-07867-x.

5. Mamtani A, Barrio AV, King TA, Van Zee KJ, Plitas G, Pilewskie M, et al. How often does neoadjuvant chemotherapy avoid axillary dissection in patients with histologically confirmed nodal metastases: results of a prospective study. Ann Surg Oncol. 2016;23:3467-74.

6. Sacre RA. Clinical evaluation of axillary lymph nodes compared to surgical and pathologic findings. Eur J Surg. 1986;12:169-73.

7. Voogd AC, Coebergh JW, can Driel OJR, Roumen RM, van Beek M, Vreugdenhil A, Crommelin MA. The risk of nodal metastases in breast cancer patients with clinically negative lymph node: a population-based analysis. Breast Cancer Res Treat. 2000;62:63-9.

8. Lanng C, Hoffmann J, Galatius H, Englel U. Assessment of clinical palpation of the axilla as a criterion for performing the sentinel node procedure in breast cancer. Eur J Surg Oncol. 2007;33:281-4.

9. Specht MC, Fey JV, Borgen PI, Cody HS III. Is the clinically positive axilla in breast cancer really a contraindication to sentinel lymph node biopsy? J Am Coll Surg. 2005;200:10-14.

10. Mumtaz H, Hall-Craggs MA, Davison T, Walmsley K, Thurell W, Kissin MW, Taylor I. Staging of symptomatic primary breast 
cancer with MR imaging. AJR Am J Roentgenol. 1997;169:417-24.

11. Luciani A, Dao TH, Lapeyre M, Schwarzinger M, Debaecque C, Lantieri L, et al. Simultaneous bilateral breast and high-resolution axillary MRI of patients with breast cancer: preliminary results. AJR Am J Roentgenol. 2004;182:1059-67.

12. Kvistad KA, Rydland J, Smethurst HB, Lundgren S, Fjosne HE, Haraldseth O. Axillary lymph node metastases in breast cancer: preoperative detection with dynamic contrast-enhanced MRI. Eur Radiol. 2000;10:1464-71.

13. Murray AD, Staff RT, Redpath TW, Gilbert FJ, Ah-See AK, Brookes JA, et al. Dynamic contrast-enhanced MRI of the axilla in women with breast cancer: comparison with pathology of excised nodes. Br J Radiol. 2002;75:220-8.

14. Weber JJ, Jochelson MX, Eaton A, Zabor EC, Barrio AV, Gemignani ML, et al. MRI and prediction of pathologic complete response in the breast and axilla after neoadjuvant chemotherapy for breast cancer. J Am Coll Surg. 2017;225:740-6.

15. Montellaro VE, Marshall J, Singer L, Hochwald SN, Chang M, Copeland EM, Grobmyer SR. Magnetic resonance imaging for axillary staging in patients with breast cancer. J Magn Reason Imaging. 2009;30:309-12.

16. Javid S, Segara D, Lotfi P, Raza S, Golshan M. Can breast MRI predict axillary lymph node metastasis in women undergoing neoadjuvant chemotherapy. Ann Surg Oncol. 2010;17:1841-6.

17. Moo TA, Edelweiss M, Hajiyeva S, et al. Is low-volume disease in the sentinel node after neoadjuvant chemotherapy an indication for axillary dissection? Ann Surg Oncol. 2018;25:1488-94.

Publisher's Note Springer Nature remains neutral with regard to jurisdictional claims in published maps and institutional affiliations. 